\title{
Ätiologie, Klassifikation, Indikation und Durchführung der operativen und konservativen Behandlung der Ellenbogensteife
}

\author{
Reinhard Meier, Jens Stehle, Cornelia Tölg, Frank Gohlke
}

\section{Zusammenfassung}

Die Steife des Ellenbogens hat vielfältige Ursachen. Neben den häufigen verletzungsbedingten bestehen vielfältige degenerative Auslöser für Kontrakturen. Die Beweglichkeit des Ellenbogens kann abhängig von der individuellen Situation durch nicht operative und operative Ansätze wiedererlangt werden. In den letzten Jahren hat sich neben den konservativen und offenen Maßnahmen zunehmend die arthroskopische Arthrolyse als Behandlungsoption etabliert. Mit diesen Optionen lässt sich vielfach die schmerzlose Funktion des Ellenbogengelenks wiederherstellen.
Aetiolgy, Classification, Indications

for and Execution of Surgical and Consevative Treatment of Stiffness of the Elbow

There are several reasons for stiffness of the elbow joint. Besides traumatic disorders there are several non-traumatic factors that can lead to contractures of the elbow. Joint motion can be restored with non-surgical and surgical interventions. Besides the non-surgical and open treatment options, arthroscopic arthrolysis has become an established method. With these options a painless, more or less complete restoration of elbow function can usually be expected.

\section{Ätiologie}

Als ursächlich für Ellenbogensteifen (Ankylosen) werden degenerative und verletzungsbedingte Auslöser unterschieden. Degenerative Veränderungen können hierbei durch rezidivierende Einblutungen, z.B. bei Gerinnungsstörungen (Hämophilie, freie Gelenkkörper oder Arthrosen und Arthritiden), auftreten. Als Verletzungen können Frakturen, Luxationen, aber auch isolierte Weichteilverletzungen zur Steife führen. Seltener bedingen angeborene Varianten und Fehlbildungen, Infektionen, Chondromatosen, Verbrennungen oder heterotope Ossifikationen, z.B. nach SHT oder Langzeitbeatmung, eine Ellenbogensteife. Mischbilder sind hierbei häufig (Tab. 1).

Das Ellenbogengelenk neigt angesichts der o.g. Auslöser auffallend häufig zur Einsteifung. Als Triebkräfte für diese Tendenz zur Steife werden die enge Ver-

OP-JOURNAL 2012; 28: 42-47

(c) Georg Thieme Verlag KG Stuttgart · New York DOI http://dx.doi.org/10.1055/s-0031-1298549 netzung der Gelenkkapsel sowohl zu den intrakapsulären Bändern als auch zu den extrakapsulären Muskeln in 3 Kompartimenten sowie die Kongruenz des Humeroulnargelenks vermutet [3].

\section{Klassifikation}

Neben der oben bereits angeführten Einteilung in Bezug auf die Ursache der Ellenbogensteife sind Klassifikationen nach Lokalisation der Pathologie und der resultierenden funktionellen Einschränkung gebräuchlich.

Je nach betroffenen anatomischen Strukturen können extraartikuläre (extrinsische) und intraartikuläre (intrinsische) Ankylosen unterschieden werden.

Im Rahmen der extrinsischen Kontraktur sind v.a. die extraartikulären Weichteile und die Gelenkkapsel betroffen. Häufig sind hier die Kollateralbänder, die ventrale Kapsel, aber auch die Mm. brachioradialis et radialis beteiligt. Bei intrinsischen Kontrakturen führen intraartikuläre Pathologien der Synovialis, des Knochens und des Gelenkknorpels in
Form von Adhäsion, Inkongruenz und Destruktion zur Steife. In der Mehrheit der Fälle treten extra- und intraartikuläre Mischformen auf. So sind z.B. infolge von Frakturen nicht nur intrinsische Gelenkinkongruenzen, sondern auch extrinsische, periartikuläre Vernarbungen und Verkalkungen regelmäßig zu beobachten.

Wenn beide Komponenten zur Bewegungseinschränkung beitragen, ist die Behandlung komplexer und die Prognose insgesamt schlechter zu sehen [13].

Kay hat in diesem Zusammenhang eine praktisch, chirurgisch orientierte Klassifikation vorgeschlagen, die zudem prognostische Kriterien berücksichtigt (Tab. 2) [11].

Eine Einteilung nach dem Ausmaß der funktionellen Störung berücksichtigt den Umfang der Bewegungseinschränkung. Hierbei gilt bei einem Bewegungsumfang (Range of Motion, ROM) in Extension/Flexion von über $90^{\circ}$ die Kontraktur als minimal, bei einem ROM von $61-90^{\circ}$ als moderat, bei $31-60^{\circ}$ als schwer und bei weniger als $30^{\circ}$ Beweglichkeit als sehr schwer [12].

Entscheidend ist dabei die Hauptrichtung der Bewegungslimitierung.

So spricht man bei einer Streckfähigkeit über $30^{\circ}$ und Beugefähigkeit unter $130^{\circ}$ von einer Extensionskontraktur. Als Flexionskontraktur wird eine Beweglichkeit von Extension unter $30^{\circ}$ und Flexion über $130^{\circ}$ bezeichnet. Die kombinierte Kontraktur zeichnet sich durch eine Streckbarkeit unter $30^{\circ}$ und Beugefähigkeit unter $130^{\circ}$ aus [12] (Abb. 1).

\section{Indikation und Durchführung der Behandlung}

Ziel der Behandlung der Ankylose ist die Wiedererlangung einer funktionellen und schmerzfreien Beweglichkeit des 
Tab. 1 Ursachen für Ellenbogensteifen.

\begin{tabular}{l} 
Traumatisch: \\
\hline - Fraktur \\
\hline - Luxation \\
\hline - Luxationsfraktur \\
\hline Degenerativ: \\
\hline - idiopathische Arthrose \\
\hline - rheumatoide Arthritis \\
\hline - Hämophilie \\
\hline - freie Gelenkkörper \\
\hline Andere: \\
\hline - Verbrennung \\
\hline - Chondromatose \\
\hline - Immobilisation \\
\hline - septische Arthritis \\
\hline - heterotope Ossifikation \\
\hline - Fehlbildung
\end{tabular}

Tab. 2 Klassifikation der Ellenbogensteifen nach Kay [11].

\begin{tabular}{ll} 
Grad I & reine Weichteilkontraktur \\
\hline Grad II & $\begin{array}{l}\text { Weichteilkontraktur mit Ossifika- } \\
\text { tionen }\end{array}$ \\
\hline Grad III & $\begin{array}{l}\text { nicht dislozierte intraartikuläre } \\
\text { Fraktur mit Weichteilkontraktur }\end{array}$ \\
\hline Grad IV & $\begin{array}{l}\text { dislozierte intraartikuläre Fraktur } \\
\text { mit Weichteilkontraktur }\end{array}$ \\
\hline Grad V & posttraumatische Knochenbrücken
\end{tabular}

Ellenbogens. Der normale Bewegungsumfang des Ellenbogens beträgt $0-0$ $145^{\circ}$ Extension/Flexion (Ext/Flex). Funktionell relevant ist hierbei für die meisten Aktivitäten des täglichen Lebens ein funktioneller Bewegungsumfang („,functional arc") von Extension/Flexion mit $0-30-130^{\circ}$ und eine Umwendbewegung in Pro-/Supination von 50-0-50 [15]. Im Einzelfall kann durchaus ein unterschiedliches Anforderungsmuster vorliegen. Insbesondere können begleitende Bewegungseinschränkungen von Schulter oder Handgelenk die Gebrauchsfähigkeit erheblich limitieren [9].

Für die Therapieplanung ist deshalb eine exakte Anamneseerhebung und Eruierung des Anforderungsprofils unerlässlich.

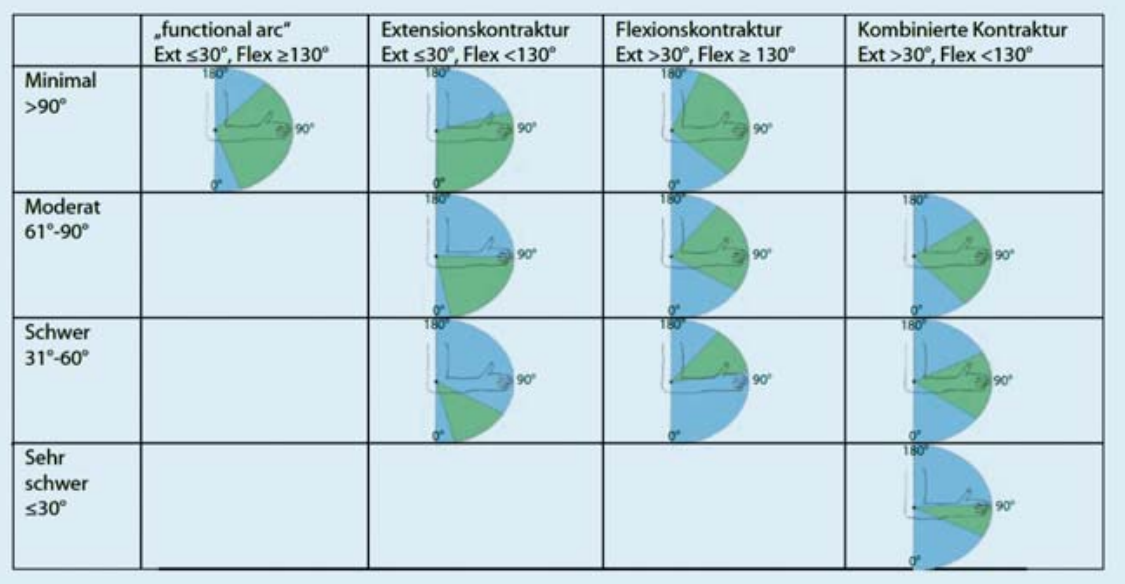

Abb. 1 Klassifikation nach Bewegungsumfang. Mögliche Bewegungsausmaße sind grün eingefärbt dargestellt [20]. (Mit freundlicher Genehmigung von Springer Science + Business Media. Aus: Stehle ], Gohlke F. [Classification of elbow stiffness and indications for surgical treatment]. Der Orthopäde 2011; 40: 282-290.)

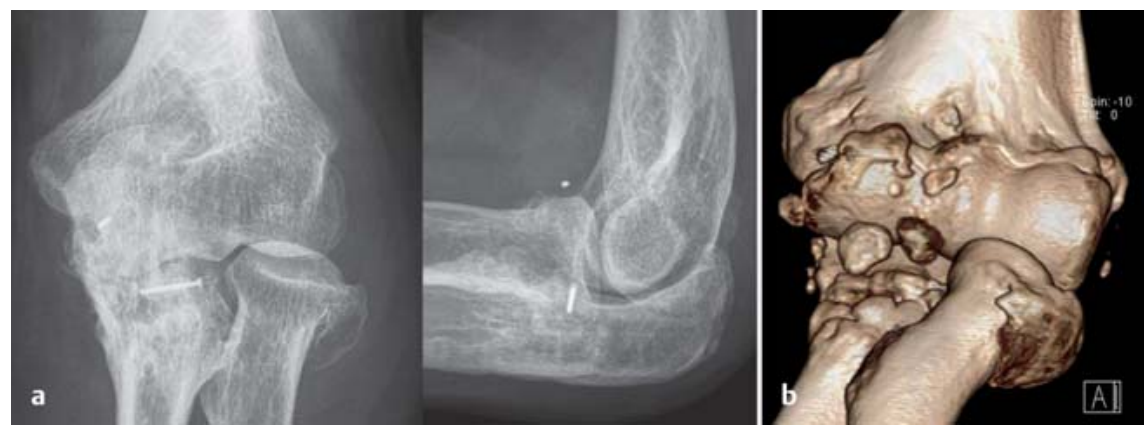

Abb. $\mathbf{2}$ a und $\mathbf{b}$ Röntgenaufnahme (a) und 3-D-CT (b) eines Patienten mit Beugehemmung. Erkennbar sind v. a. Osteophyten, Implantatreste und freie Gelenkkörper.

Als weitere Basis zur Wahl der adäquaten Behandlungsmethode dient die exakte klinische Untersuchung der oberen Extremität. Hierbei sind neben der Ermittlung der passiven Gelenkfunktion und Stabilität eine ausreichende neurologische und muskuläre Funktion zu verifizieren. Ohne diese ist eine dauerhafte adäquate Ansteuerung und Gebrauchsfähigkeit des erzielten Bewegungsumfangs unwahrscheinlich.

Für die definitive Behandlungsfestlegung ist die radiologische Diagnostik essenziell. Konventionelle Röntgenaufnahmen im anterior-posterioren und seitlichen Strahlengang bilden die meisten knöchernen Veränderungen wie Arthrosen, Osteophyten, ektope Ossifikationen oder Fehlstellungen ab. Sie können durch Schnittbildgebung (Computertomografie und Magnetresonanztomografie) ergänzt werden (Abb. 2). Diese liefern zusätzliche wichtige Informationen über die Lokalisation und Beschaffenheit der zugrunde liegenden Pathologie. Während die Computertomografie Vorteile in der detaillierten Abbildung knöcherner Strukturen bietet, hat die Kernspintomografie Vorzüge in der Darstellung von Weichteilprozessen und in der Abbildung von knorpeligen Gelenkkörpern. Sollte eine operative Intervention geplant werden, sehen wir die CT mit 3-DRekonstruktionen v.a. zur exakten Visualisierung der ulnaren Veränderungen als unerlässlich an.

Prinzipiell erfordert jede funktionsverbessernde Maßnahme einen erheblichen Aufwand für den Patienten und eine entsprechende Bereitschaft der aktiven Mitarbeit (Compliance). Zumal die Erfolgsaussichten sowohl der konservativen als auch der operativen Behandlung der Ellenbogensteife durchaus differenziert $\mathrm{zu}$ betrachten sind.

Es stehen operative und konservative Behandlungsoptionen zur Verfügung, wobei zwar ein rein konservativer Ansatz möglich ist. Eine operative Intervention bedarf jedoch immer auch einer begleitenden konservativen Therapie. 
Tab. 3 Kontraindikationen für eine operative Arthrolyse.

- mangelnde Compliance des Patienten

- bestimmte zentralnervöse Störungen (Spastik, verminderte kognitive Aufnahmefähigkeit)

- lokale Infektion

- Haut-/Weichteilschäden

Die nicht operativen Ansätze beinhalten vor allem physiotherapeutisch unterstützte Übungsbehandlungen und Mobilisierungen [1] sowie statische und dynamische Orthesen und Schienenbehandlungen [6,19,24]. Diese Maßnahmen können bereits als alleinige Therapie eine deutliche Funktionsverbesserung erreichen und sollten demzufolge in der Regel einer operativen Intervention vorausgehen. Bei Kontraindikationen für eine operative Intervention bleiben sie ohne dies die alleinige Therapiemodalität (Tab. 3). Die Ergebnisse der rein konservativen Ansätze sind schlechter bei Patienten mit intraartikulärer Ursache der Steife.

Bei bleibenden, ausgeprägten funktionellen Defiziten und dringendem Wunsch des Patienten zur Verbesserung der Situation ist ein operativer Eingriff zur Gelenklösung angezeigt. Prognostisch günstige Faktoren für ein operatives Vorgehen sind dabei behandelbare intrinsische/intraartikuläre Pathologien und knöchern bedingte Bewegungseinschränkungen.

Zur Operationsplanung muss eine exakte Analyse der zugrunde liegenden Ursache, der betroffenen Strukturen und der individuellen Bedürfnisse vorliegen.

Oft sind mehrere Pathologien an der Entstehung einer Ellenbogensteife beteiligt. Jede für sich muss adäquat adressiert werden.

Auch der Zeitpunkt für eine operative Therapie muss sorgfältig überlegt werden. Beim Vorliegen einer posttraumatischen Ellenbogensteife sollte 6-12 Monate nach dem initialen Trauma (chronische Phase) eine Operation geplant werden. Verletzungen müssen konsolidiert, Frakturen verheilt und die osteoinduktive Potenz kontrakter Strukturen bereits wieder reduziert sein. Ein längeres zeitliches Intervall reduziert die Erfolgsaussichten wieder $[2,8,13]$. In der Zwischenzeit (subakute Phase) sollte die konservative Therapie optimiert werden, um eine bestmögliche funktionelle Ausgangssituation zu schaffen bzw. eine Intervention gegebenenfalls sogar ganz zu vermeiden [20].

Die Planung des Zugangs orientiert sich an der Lokalisation der Pathologie und den beteiligten Kompartimenten. Die häufig betroffenen ventralen und dorsalen Strukturen sind in der Regel gut über einen dorsoradialen Zugang (,lateral column procedure“) erreichbar. Isolierte ventrale Pathologien können auch über einen vorderen Zugang entfernt werden $[7,21]$. Aufgrund der im Zugangsbereich befindlichen neurovaskulären Strukturen und des $\mathrm{M}$. brachialis, der ohnehin zu Ossifikationen neigt, ist dieser Zugang jedoch komplikationsträchtig.

Isolierte ulnare Ursachen der Steife, z.B. die Verkürzung des Kollateralbands oder Erkrankungen des N. ulnaris, können über einen medialen Zugang sinnvoll gelöst werden.

Der N. ulnaris sollte insbesondere bei Streckkontrakturen bereits in der Planung Beachtung finden. Es besteht die Gefahr, dass es nach Wiedererlangen der Beugefähigkeit des Ellenbogens durch Verwachsungen und Verklebung zu Zugschäden des Nervs kommt. Es sollte deshalb bei Lösung von ausgeprägten Streckkontrakturen immer eine Ventralverlagerung des $\mathrm{N}$. ulnaris in Betracht gezogen werden.

Eine weitere Methode, die auch arthroskopisch angewandt werden kann, beinhaltet die Fensterung der Fossa olecrani von dorsal (Outerbridge-Kasiwagi-OP) [10]. Dadurch können sowohl anteriore als auch posteriore Gelenkkörper erreicht und ein Impingement der Processi olecrani und coronoidei verhindert werden.

Eine tetrafokale Arthrolyse unter temporärer Ablösung der Seitenbänder und Verlagerung des Nervs über einen längeren streckseitigen Zugang ist bei ausgedehnten heterotopen Ossifikationen, die ventral und dorsal liegen und sowohl das ulnare als auch radiale Kompartiment und den Sulcus ulnaris betreffen, empfehlenswert, da hierdurch das Trauma für den Weichteilmantel reduziert werden kann [20].

Einfluss auf die Zugangswahl nehmen in nicht unerheblichem Ausmaß auch Narben vorangegangener Eingriffe.
Die Lagerung ist prinzipell auf dem Rücken, der Seite und dem Bauch möglich. Wir bevorzugen für Ellenbogeneingriffe die standardisierte Seitenlagerung (Abb. 3).

\section{Offene Arthrolyse}

Je nach in der Planung gewähltem Zugangsweg erfolgt die Präparation unter sorgfältiger Schonung der neurovaskulären Strukturen. Besondere Beachtung findet hierbei der N. ulnaris, der gegebenenfalls auch über eine zusätzliche mediale Inzision dargestellt wird. Meist ist im Verlauf der Operation dessen Neurolyse und ggf. subkutane Verlagerung indiziert, vor allem, um Dehnungsschäden nach Erweiterung des Bewegungsumfangs des Ellenbogens zu vermeiden. Je nach Befund erfolgen dann eine möglichst vollständige Entfernung von Ossifikationen, Briden und Narben unter Darstellung des ulnaren oder radialen Kapsel-Band-Apparats, bis das Gelenk frei einsehbar ist. Blockierende Exophyten werden ebenso, wie evtl. vorhandene Implantate, entfernt (Abb. 4). Sollte noch kein ausreichender Bewegungsumfang erreicht sein, wird die Kapsel in- bzw. exzidiert. Nach jedem Schritt wird das Gelenk vorsichtig zunehmend gebeugt und gestreckt sowie umgewendet.

Falls die Seitenbänder ossifiziert sind, sollten diese in gleicher Sitzung mittels einer lokalen Bandplastik ersetzt werden. Bei persistierender intraoperativ bestehender Instabilität, die durch Bandrekonstruktionen alleine nicht beherrschbar ist, kann ein Bewegungsfixateur verwendet werden [17]. Dabei wird für 6-8 Wochen eine konstante Distraktion über den gesamten Bewegungsumfang gehalten, bei gleichzeitiger Einhaltung des anatomisch korrekten Rotationszentrums [23].

\section{Arthroskopische Arthrolyse}

Indikationen für arthroskopische Arthrolysen sind gering bis mäßig ausgeprägte Gelenksteifen mit einem ROM von über $60^{\circ}$ bei rein intrinsischen Kontrakturen, die z. B. durch intraartikuläre Briden, Osteophyten, Osteochondrosis dissecans (OD) oder freie bzw. adhärente Gelenkkörper verursacht werden.

Relevante extraartikuläre Ossifikationen oder Gelenkdeformierungen gelten als Kontraindikation für ein rein arthroskopisches Vorgehen. Ebenso gilt ein schweres Flexionsdefizit $\left(<100^{\circ}\right)$ als Indikation 

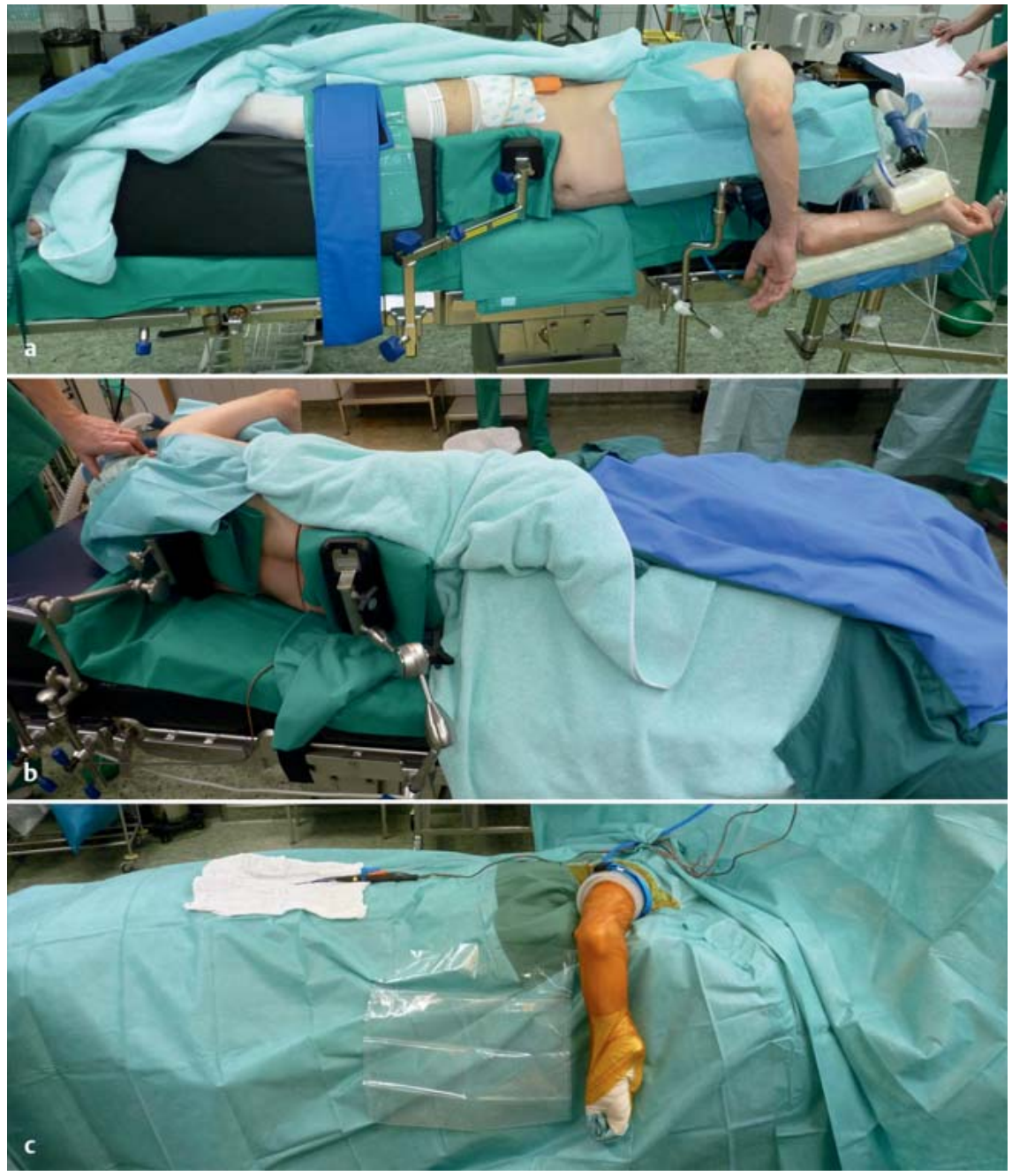

Abb. 3 a bis c Seitlagerung zur arthroskopischen und offenen Ellenbogenarthrolyse. Der Patient wird auf der kontralateralen Seite gut unterpolstert und mit Stützen am Becken und Oberkörper gelagert, sodass intraoperatives Kippen des Tisches möglich ist (a, b). Der Arm wird nach Desinfektion frei beweglich abgedeckt und eine sterile Blutsperremanschette angelegt (c).

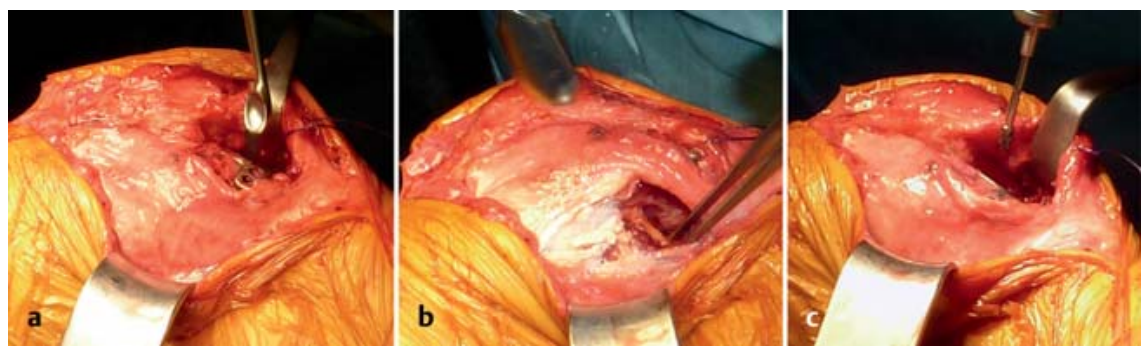

Abb. 4 a bis c Offene Arthrolyse. Osteophyten, Narben und interponierendes Gewebe werden mit scharfem Löffel (a), Meißel und Raspatorium (b) oder einer Hochfrequenzfräse (c) vorsichtig gelöst und abgetragen.

für einen offenen Zugangsweg, da hier meist der N. ulnaris dekomprimiert oder verlagert werden muss.

Durch die Entwicklung der endoskopischen Techniken unter Verwendung spezieller Kurzschaft-Spülsysteme besteht beste Indikation für ein arthroskopisches Kapselrelease dar [4]. Dennoch bleibt sie ein technisch anspruchsvoller Eingriff mit dem Risiko von schweren Verletzungen von peripheren Nerven, Gefäßen und Knorpel [22].

Um eine Vergrößerung der Distanz zwischen Humerus und neurovaskulären Strukturen zu erreichen, wird das Ellenbogengelenk zunächst im Soft Spot über eine Nadel mit 20-30 ml Ringer- oder Kochsalzlösung aufgefüllt. Unterbleibt die Aufweitung des Gelenks nach Instillation der Flüssigkeit um $20-30 \mathrm{ml}$, z.B. bei kapsulärer Steife, erhöht sich die Gefahr von Komplikationen wie Nervenund Gefäßverletzung. Nach Auffüllung des Gelenks wird in der Regel das proximale anteromediale Portal gesetzt. Dies befindet sich typischerweise $2 \mathrm{~cm}$ proximal des medialen Epikondylus und 1-2 $\mathrm{cm}$ anterior des Septum intermusculare mediale. Beachtung muss hierbei die Lage des N. ulnaris finden. Transposition oder ventrale Subluxation des Nervs stellen Kontraindikationen für dieses Portal dar. Nach der Hautinzision wird stumpf über einen Wechselstab oder Trokar vorgegangen, um den $\mathrm{N}$. cutaneus antebrachii medialis, der sich nahe am Portal befindet, zu schonen. Eine Kanüle für die Optik wird 2-3 cm anterior des Septum intermusculare mit dem Ziel, das Gelenk am medialsten Punkt zu eröffnen, positioniert. Nach Etablierung des anteromedialen proximalen Portals ergibt sich ein Blick auf den anterioren radiohumeralen Gelenkabschnitt.

Zusätzlich wird $2 \mathrm{~cm}$ proximal des lateralen Epikondylus, anterior des suprakondylären Raumes, das proximale anterolaterale Portal angelegt. Es wird, um Läsionen des $\mathrm{N}$. radialis zu vermeiden, über einen Wechselstab in der „InsideOut-Technik“ installiert. Das Portal wird mit einer Kanüle gesichert. Intraartikuläre Verklebungen werden gelöst. Dann wird die vordere Kapsel vom Humerus gelöst. Mit dem Shaver werden Synovia, Adhäsionen, freie Gelenkkörper und anteriore Osteophyten abgetragen.

Die anteriore Kapsulotomie sollte mit dem Punch unter Sicht erfolgen, um neurovaskuläre Verletzungen durch ein $\mathrm{zu}$ ausgeprägtes und unkontrolliertes Débridement der Kapsel mit dem oszillierenden Shaver zu vermeiden. Das Abschieben der Gelenkkapsel vom Humerus kann mit dem scharfen Raspatorium erfolgen. Die Kapsel wird von lateral nach medial hin inzidiert. Zur Vervoll- 
ständigung der Kapsulotomie wird ein Wechsel der Instrumente auf das anteromediale Arbeitsportal empfohlen, sodass die Oberfläche des M. brachialis zur Darstellung kommt.

Über ein posterolaterales Portal, $2 \mathrm{~cm}$ proximal des Olekranons, lateral der Trizepsinsertion, wird die posteriore Kapsel vom Humerus abgelöst und so ein Release der Kapsel bis zum medialen Rand der Fossa olecrani vorgenommen. Dorsale Osteophyten werden mit dem scharfen Löffel, der Kugel- oder Walzenfräse abgetragen.

Durch vorsichtige Bewegung des Ellenbogengelenks werden verbleibende Verklebungen nach Entfernen der Instrumente mobilisiert. Die Inzisionen werden vernäht und steril abgedeckt. Die Blutsperre wird nach Anlage eines Druckverbands entfernt (Abb. 5 und 6).

\section{Andere Verfahren}

\section{Endoprothese}

Der prothetische Ersatz ist eine sinnvolle Behandlungsmethode bei Bewegungseinschränkungen aufgrund einer Arthrose oder postraumatischen Gelenkzerstörung. Bei knöchern durchbauter Ankylose ist der endoprothetische Ersatz jedoch mit einer hohen Komplikationsrate verbunden [16].

Insbesondere bei jüngeren und aktiven Patienten unter 60 Jahren ist mit einer frühzeitigen Lockerung zu rechnen. So ist die Ellenbogenprothetik in der Regel dem älteren Patienten, der voraussichtlich den Ellenbogen nicht mehr stark beanspruchen wird, vorbehalten.

\section{Resektions-Interpositions-Arthroplastik}

Bei jüngeren Patienten ist eher eine Interpositionsarthroplastik mit Fascia lata [14] oder Dermis [5] indiziert. Dabei werden die o.g. Gewebe zwischen die Gelenkpartner eingeschlagen und fixiert. Als Indikation gilt ein junger Patient mit kongruentem Gelenk. Kontraindikationen sind deutlicher Knochenverlust, Infektionen oder Instabilitäten. Verbleibt nach dem Release eine signifikante Instabilität, ist eine Kombination mit Bewegungsfixateuren sinnvoll [18]. Eine reine Resektionsarthroplastik liefert demgegenüber aufgrund der erzeugten Instabilität und fortschreitender Knochenresorption langfristig oft unbefriedigende Ergebnisse.

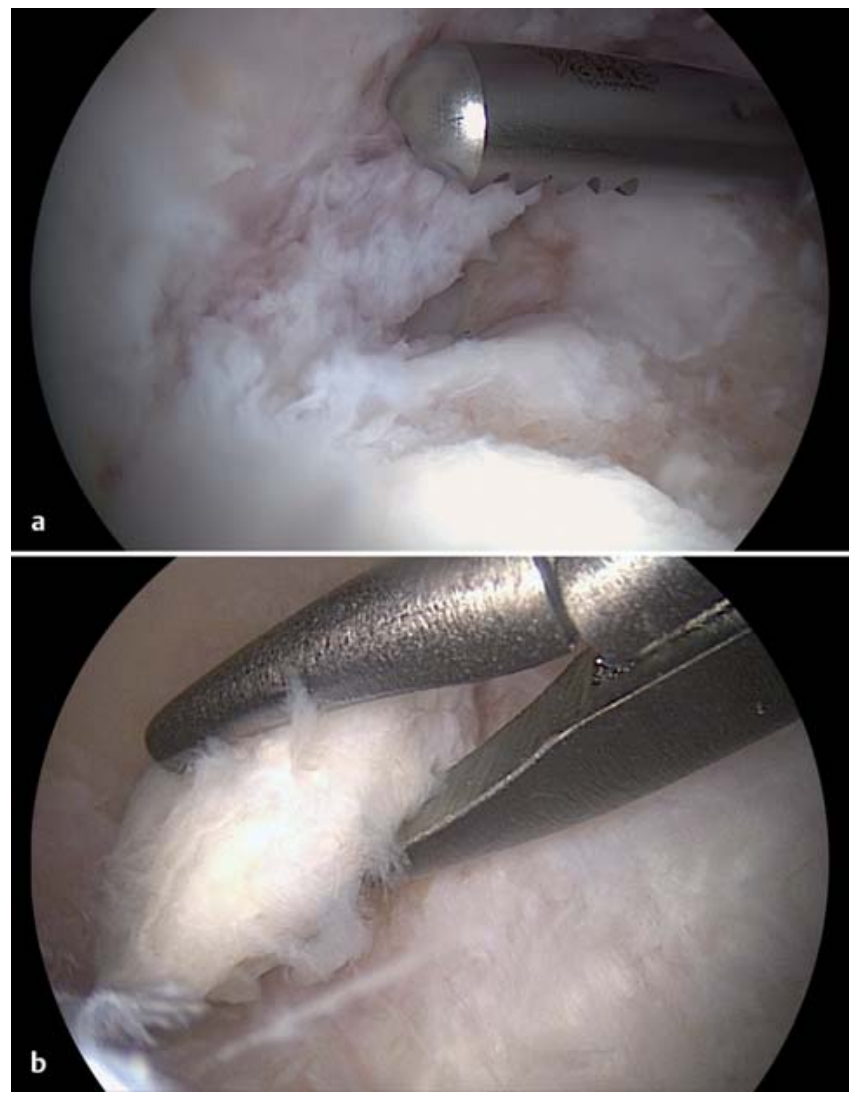

Abb. $5 \mathrm{a}$ und $\mathrm{b}$ Arthroskopische Arthrolyse. Mit dem Shaver werden Adhäsionen gelöst (a). Freie Gelenkkörper und Ossifikationen werden entfernt (b)

\section{Arthrodese}

Eine Arthrodese ist gelegentlich eine letzte Rückzugsmöglichkeit v.a. bei chronischen Infektionen und persistierenden Schmerzen sowie nach fehlgeschlagener TEP-Versorgung. Voraussetzung ist das Vorliegen von ausreichend knöcherner Substanz. Die Stabilität und Schmerzfreiheit wird hierbei mit einer erheblichen Funktionseinschränkung erreicht.

\section{Nachbehandlung}

Unsere Nachbehandlung nach Arthrolysen des Ellenbogens wird individuell abgestimmt. In der Regel wird direkt nach dem Eingriff der Arm in einer dorsalen Oberarmgipsschiene mit sorgfältiger Polsterung abhängig vom intraoperativ erzielten Bewegungsumfang in mittlerer Streckung von $20-45^{\circ}$ gelagert.

Nach Entfernung der Drainagen wird frühzeitig funktionell mobilisiert.

Im Rahmen der perioperativen Narkose können bei sehr ausgeprägten Gelenksteifen 2 Gips- oder Kunststoffschalen in der individuellen, maximal erreichbaren Streckung und Beugung bei mittlerer Pro-/Supination angefertigt werden.
Hierin wird der Arm ab dem 1. postoperativen Tag in 2-stündiger Frequenz umgelagert.

Ebenfalls ab dem 1. postoperativen Tag wird zur Prophylaxe von heterotopen Ossifikationen ein nicht steroidales Antiphlogistikum (z.B. Indometacin) verabreicht. Alternativ ist auch eine Bestrahlung des Ellenbogengelenks innerhalb der ersten 3 postoperativen Tage zu erwägen.

In der 1. postoperativen Phase werden Plexuskatheter zur Schmerzausschaltung verwendet. Besondere Vorsicht ist hierbei geboten, um neben einschnürenden bzw. drückenden Verbänden progressive Dehnungsparesen v.a. des N. ulnaris nicht zu übersehen. Es sollten Unterbrechungen der Medikation zur Kontrolle eingelegt werden [20].

Zusammenfassend können vielfältige intrinsische und extrinsische Faktoren ursächlich für Ellenbogensteifen sein. Entsprechend ist die Therapie individuell abgestimmt. Sie beinhaltet neben den konservativen Verfahren unterschiedliche Varianten der arthroskopischen und offenen Arthrolyse, den Bewegungsfixateur oder endoprothetischen Ersatz. Orientiert sich das gewählte Verfahren an 


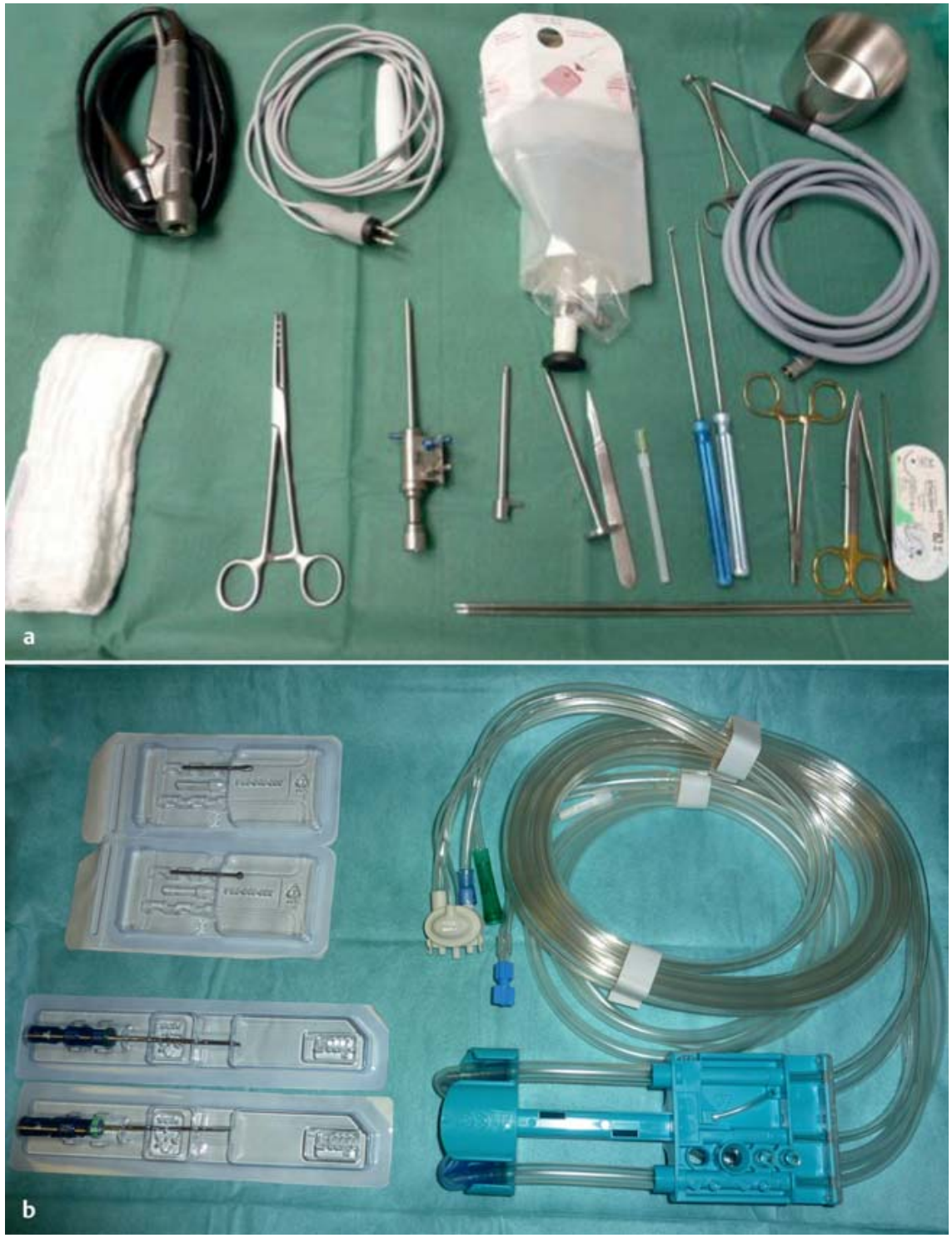

Abb.6a und b Instrumententisch zur arthroskopischen Arthrolyse. Von links: a oben: Shaver, bipolares Koagulationssystem, Optik, Lichtkabel mit Tuchklemme und Schale; unten: Bauchtuch, Klemme, Schleusen, Trokar, Skalpell, Kanüle, Tasthaken, Disektor, Nadelhalter, Schere, Pinzette, Faden, mind. 2 Wechselstäbe. b Fräsen- und Shaveraufsätze sowie Schlauchmodul für druckund volumenkontrolliertes Pumpensystem. Nicht abgebildet: kleiner Punch und Fasszange.

der Ätiologie, den betroffenen Kompartimenten und Strukturen sowie den individuellen Bedürfnissen des Patienten, kann die Funktionalität des Ellenbogens wiederhergestellt werden.

\section{Literatur}

Bonutti PM, Windau JE, Ables BA et al. Static progressive stretch to reestablish elbow range of motion. Clin Orthop Relat Res 1994; 303: $128-134$

2 Breitfuss H, Muhr G, Neumann K et al. [Arthrolysis of post-traumatic stiff elbow. Which factors influence the end result]. Unfallchirurg 1991; 94: 33-39
${ }^{3}$ Cooney W, Morrey B. Contractures of the Elbow. Philadelphia: W. B. Saunders; 1993

${ }^{4}$ Ditsios KT, Werner BS, Yamaguchi K. [Arthroscopic capsular release of the elbow]. Orthopade 2011: 40: 296-302

${ }^{5}$ Froimson AI, Silva JE, Richey D. Cutis arthroplasty of the elbow joint. J Bone Joint Surg [Am] 1976; 58: 863-865

${ }^{6}$ Green DP, McCoy H. Turnbuckle orthotic correction of elbow-flexion contractures after acute injuries. J Bone Joint Surg [Am] 1979; 61: 1092-1095

7 Henry A. Extensile Exposures. Edinburgh: Livingstone Ltd.; 1957

8 Judet J, Judet $H$. [Arthrolysis of the elbow]. Acta Orthop Belg 1975; 41: 412-413

${ }^{9}$ Kapandji I. The Physiology of the Joints. New York: Churchill Livingstone; 1982
${ }^{10}$ Kashiwagi D. Arthrolysis of the Elbow. In: Kashiwagi D, ed. Proceedings of the international Seminar Elbow Joint. Kobe: Elsevier Science; 1985

${ }^{11}$ Kay N. Arthrolysis of the posttraumatic stiff Elbow. In: Stanley D, Kay NR, eds. Surgery of the Elbow. Practical and scientific Aspects. London: Arnold; 1998: 228-234

12 Mansat P, Morrey BF. The column procedure: a limited lateral approach for extrinsic contracture of the elbow. J Bone Joint Surg [Am] 1998; 80: 1603-1615

${ }^{13}$ Morrey BF. Post-traumatic contracture of the elbow. Operative treatment, including distraction arthroplasty. J Bone Joint Surg [Am] 1990; 72: 601-618

${ }^{14}$ Morrey BF. Distraction arthroplasty. Clinical applications. Clin Orthop Relat Res 1993: 293: 46-54

${ }^{15}$ Morrey BF, Askew LJ, Chao EY. A biomechanical study of normal functional elbow motion. J Bone Joint Surg [Am] 1981; 63: 872-877

16 Peden JP, Morrey BF. Total elbow replacement for the management of the ankylosed or fused elbow. J Bone Joint Surg [Br] 2008; 90: 1198-1204

17 Pennig D, Heck S, Mader K. [Distraction arthroplasty for treatment of posttraumatic elbow stiffness]. Orthopade 2011; 40: 329-338

${ }^{18}$ Regan WD, Reilly CD. Distraction arthroplasty of the elbow. Hand Clin 1993; 9: 719-728

${ }^{19}$ Shewring DJ, Beaudet M, Carvell JE. Reversed dynamic slings: results of use in the treatment of post-traumatic flexion contractures of the elbow. Injury 1991; 22: 400-402

20 Stehle J, Gohlke F. [Classification of elbow stiffness and indications for surgical treatment]. Orthopade 2011; 40: 282-290

${ }^{21}$ Urbaniak JR, Hansen PE, Beissinger SF et al. Correction of post-traumatic flexion contracture of the elbow by anterior capsulotomy. J Bone Joint Surg [Am] 1985; 67: 1160-1164

22 Van Zeeland NL, Yamaguchi K. Arthroscopic capsular release of the elbow. J Shoulder Elbow Surg 2010; 19 (Suppl.): 13-19

${ }^{23}$ Volkov MV, Oganesian OV. Restoration of function in the knee and elbow with a hinge-distractor apparatus. J Bone Joint Surg [Am] 1975; 57: 591-600

24 Zander CL, Healy NL. Elbow flexion contractures treated with serial casts and conservative therapy. J Hand Surg Am 1992; 17: 694697

Priv.-Doz. Dr. med. Reinhard Meier Oberarzt

Cornelia Tölg

Ltd. OP-Schwester

Bereich Schulterchirurgie

Prof. Dr. med. Frank Gohlke

Chefarzt

Klinik für Schulterchirurgie

Salzburger Leite 1

97616 Bad Neustadt a.d. Saale

reinhard.meier@handchirurg.org

\section{Dr. med. Jens Stehle}

Sportklinik Ravensburg

Bachstraße 52

88214 Ravensburg 